\title{
A Study on the Effect of Cognitive Style in the Field of STEM on Collaborative Learning Outcome
}

\author{
Hsin-Ke Lu and Peng-Chun Lin
}

\begin{abstract}
Due to the rapid development of the Internet and information technology, schools of all levels around the world pay special attention to the talent cultivation in the field of science, technology, engineering and mathematics (STEM). In STEM education, the cultivation of knowledge and skills plays an important part, and teamwork and problem-solving abilities are critical soft skills for the future talent.

In teaching practice, individual differences have long been a critical issue and an important reference indicator for adaptive teaching. This study adopted a hidden figure test to investigate the difference in learning outcome and activeness among learners with different cognitive styles in the STEM collaborative learning activities. The hidden figure test based on the theory of field-dependent and field-independent cognitive styles, proposed by Witkin (1962), was originally developed by Messick (1746) and adapted by Wu (1974).
\end{abstract}

Index Terms-Cognitive style, collaborative problem solving, STEM, simulation learning, field independence.

\section{INTRODUCTION}

Due to the rapid development of the Internet and information technology, schools of all levels around the world pay special attention to the talent cultivation in the field of science, technology, engineering and mathematics (STEM). The purpose of STEM education is not limited to teaching the separate and discrete knowledge of science, engineering, and mathematics. Instead, the purpose of STEM education is to cultivate students' innovative spirit and practical ability by integrating knowledge and spirit of each subject and applying them to real life. Therefore, in STEM education, the cultivation of knowledge and skills plays an important part, and teamwork and problem-solving abilities are critical soft skills for the future talent.

In the process of cultivating learners' practical ability and helping learners learn procedural knowledge, it is important to provide them with equipment and tools. However, in practice, considering funds, budget, space, equipment maintenance, etc., it is somewhat difficult for the school authorities to provide these facilities for learners. Therefore, the use of information technology to provide learners with simulated environment plays a vital role to assist the learning of procedural knowledge [1], [2].

In terms of cultivating learners' teamwork ability, teachers use various teaching strategies to assist the proceeding of teaching activities, such as collaborative learning, project-based learning, and problem-based learning. In order

Manuscript received October 25, 2016; revised July 3, 2017.

The authors are with the Chinese Culture University, Taipei, Taiwan (e-mail: sklu@sce.pccu.edu.tw). to guide learners to learn actively and initiatively, most teaching strategies are based on collaborative learning. Collaborative learning can prompt learners with different learning characteristics to participate in learning actively and initiatively through group activities and enhance their learning outcome. Therefore, the present study adopted collaborative problem solving (CPS) to teach STEM and to train learners' collaborative learning and problem-solving abilities.

On the other hand, in teaching practice, individual differences have long been a critical issue and an important reference indicator for adaptive teaching. The indicators of individual differences adopted by the studies on general fields mainly included gender, age, education background, and other personal characteristics; in contrast, those adopted by the studies on education consist of the difference in individual's cognition, such as learning style, teaching style, perceived teaching, and so on [3].

In addition, previous research also found that different cognitive styles may influence students' learning behavior [4]-[6]. Therefore, the current study adopted a hidden figure test [7] to investigate the difference in learning outcome and activeness among learners with different cognitive styles in the STEM collaborative learning activities. The hidden figure test based on the theory of field-dependent and field-independent cognitive styles, proposed by Witkin (1962), was originally developed by Messick (1962) and adapted by $\mathrm{Wu}(1974)$.

\section{LITERATURE REVIEW}

\section{A. Cognitive Style}

The discussion of cognitive style originated in the twentieth century. Cognitive style refers to individuals' habitual patterns of behavior in the cognitive process. In addition, cognitive style, a kind of individual differences having cultivated mostly since childhood, indicates the development of perception, memory, attitude in the problem-solving process, and ways of expression. However, cognitive style was not significantly related to intelligence quotient [8].

Researchers of the previous studies divided cognitive style into different categories based on different theories and conceptualization. Based on the concept of cognitive control, Gardner, Holzman, Klein, Linton and Spence (1959) discussed the difference between leveling style and sharpening style [9]. On the basis of the concept of psychological differentiation theory, Witkin (1964) proposed field-independent (FI) and field-dependent (FD) [4]. Based 
on the concept of sensory modalities, Riessman (1964) proposed visual, auditory, and kinesthetic cognitive style [10]. Kagan (1965) adopted the theory of conceptual tempo to distinguish reflective style and impulsive style [11]. Gregorc (1977), in accordance with the attitude of dealing with information and chores, proposed concrete sequential and abstract random, abstract sequential, and concrete random [12].

Among all the categorizations of cognitive style, the theory of field independence (FI) and field dependence proposed by Witkin (1964) is the most adopted framework in the academic studies [4]. Learners of field dependence tend to rely on hints and information from others to make a decision and play a passive role in the process of learning. On the other hand, learners of field independence tend to count less on obvious or salient hints to make a decision and play an active role and make more assumptions in the process of learning.

Previous studies have found that there was a significant difference in problem solving between learners of field independence and those of field dependence [13], [14]. Some studies have shown that field-dependent learners have significantly different academic performance from field-independent learners [15], [16]. Additionally, in the research on collaborative learning, scholars found that a heterogeneous group mixed with field-independent and field-dependent learners performed significantly better than groups with other grouping methods [17].

Therefore, the present study used the theory of field independence and field dependence proposed by Witkin (1962) and adopted a hidden figure test developed by Messick (1962) and adapted by Wu (1974) as a measuring tool to assess cognitive style [7]. The administration of the hidden figure test can be divided into two phases, in each of which the participants were required to answer questions about 16 complex geometric figures in 10 minutes. Each complex geometric figure comprises one of the five basic figures and decorated with extra lines. The participants were required to recognize the basic figure from the complex geometric figure. Fig. 3 shows an example of the test. The total number of the questions is 32 . The scoring is that the number of correct answers minuses the number of wrong answers. To adjust the guessing effect, the obtained score was divided by 4 . The total score is 32 . If one obtains more points, he or she may be more inclined to field independence; if one obtains fewer points, he or she may be inclined to field dependence.

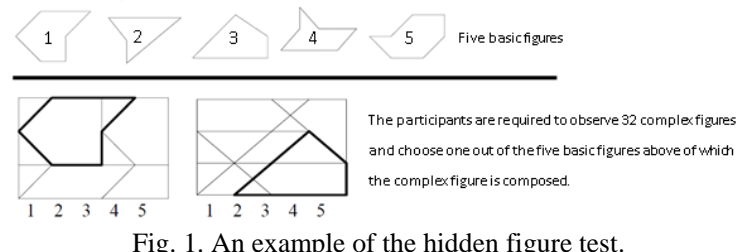

\section{B. Collaborative Problem Solving (CPS)}

In the field of STEM, much knowledge needs acquiring by doing and by trying to remove the obstacle, i.e., the process of problem solving. Therefore, problem-solving strategies are frequently applied to the teaching methods in this field to learn the procedural knowledge [18]-[21]. In addition, many studies have shown that collaborative learning can improve effectively academic performance of learners of all age. Thus, Nelson (1999) proposed collaborative problem solving (CPS), which integrates collaborative learning and problem-based learning and emphasizes on collaborative learning in authentic environment. Furthermore, in collaborative problem solving, learners are encouraged to learn by doing and to be an active learner. Independent thinking and problem solving abilities are accentuated as well [22].

What's more, in collaborative problem solving, besides problem solving skills, it is important to cultivate learners' cooperative and communicative abilities. In a heterogeneous group, one has different or limited ability and viewpoints, so group members should collaborate with one another and communicate and negotiate to agree on the most appropriate solution. Previous studies also indicated that collaborative problem solving can assist students to learn and promote their learning performance [2], [23]. Consequently, the present study follows the nine steps of collaborative problem solving to teach and use Facebook as a platform to investigate and record learners' learning outcome and process of discussion.

\section{Simulation Learning}

In the field of STEM, simulation learning plays an important role and has a crucial function in learning [1]. Simulation learning is a kind of teaching and learning strategy which provides simulated realistic situation and environment for learners to practice [24]. This learning strategy allows learners to practice and to be trained in a safer and lower-cost environment, but still immerse in an environment similar to the realistic one [25]. Therefore, nowadays simulation learning has been applied to cultivate students' or employees' skills in education institution and enterprises [26], [27]. For example, in the Department of Information Engineering, the skill of network troubleshooting is an important learning topic in the course of Computer Network. However, due to limited funds and equipment, learners may not have the chance to use the real materials. Hence, an Internet simulator can overcome the problem and be a vital tool which can assist teaching for teachers.

In the current study, the participants were the learners in the course of Network Management, the contents of which are the introduction to network and Network troubleshooting. Therefore, the study adopted Packet Tracer developed by Cisco System as the teaching simulator in the class. Packet Tracer is a program for the network concept and the network configuration of routers and switches. By an interface with graphics and instant feedback, students can design and simulate network transmission in the real situation and learn the abstract knowledge which is hard to be observed. Previous studies also pointed out that Packet Tracer-assisted teaching can provide students a more systematic and logical learning environment and improve their learning interests and learning outcome [3], [28].

\section{RESEARCH METHOD}

This study adopted a quasi-experimental design. The 
objective of the teaching activities was to develop learners' network concept and troubleshooting. In addition, the teaching activity was designed by using collaborative problem solving strategies to investigate the influence of cognitive style on collaborative learning performance in the field of STEM. This study recruited 37 participants, 7 females $(19 \%)$ and 30 males $(81 \%)$, who took the course of Network Management in a university in Taiwan.

\section{A. Experimental Design}

The total period of the course of Network Management was 18 weeks with two hours for each week. From week 1 to 15 , the class focused on teaching the basic concept of networks and the basic network troubleshooting skills. In week 16, a pretest on network concept and troubleshooting and a scale on field-independent/dependent cognitive style were administered. According to the results of the pretest and the scale, the participants were divided into heterogeneous groups. In week 17, the teaching activity for the present study was performed and post-test was given. In week 18, a review of teaching activity was carried out. Fig. 2 shows the procedure of the teaching activity.

The teaching activity in this research was done in groups. Each group was composed of 4 to 5 learners, one of whom was the group leader to check each group's learning progress. Each learner could retrieve the exercises of simulated network troubleshooting and do the exercises on their computer. After each group leader received a guiding worksheet, the members of each group could start discussing with one another on a collaborative learning discussing platform, a fan page for each group in isolation on Facebook. Fig. 3 shows the operating interface of the simulated platform for learners to set up a network and troubleshooting.

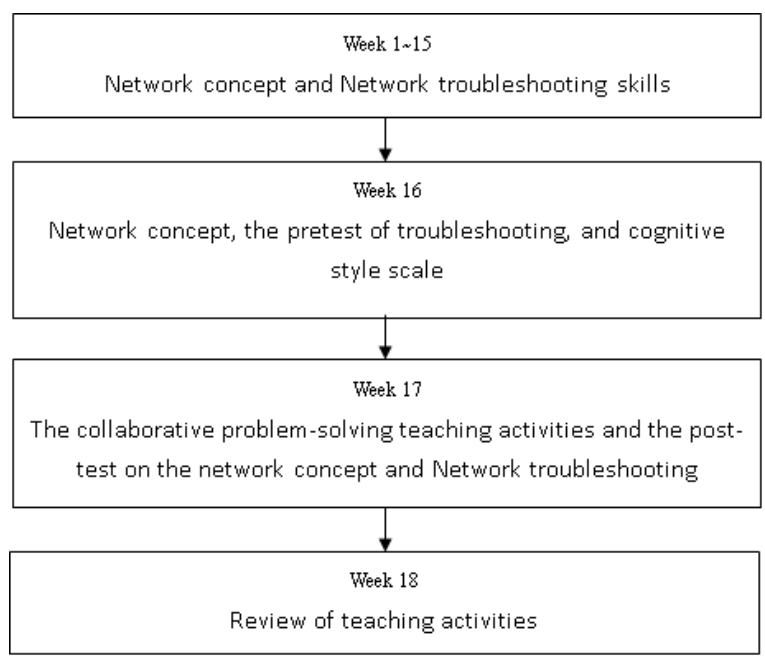

Fig. 2. The procedure of the teaching activities.

\section{B. Research Instruments}

The research instruments in this study included a network concept and troubleshooting test, a cognitive style test, a guiding worksheet, and a simulated operation problem.

- A network concept and troubleshooting test: this test contains 8 multiple choice questions, 10 points for each question, and an essay question, 20 points the question, in order to test learners' network concept and basic network troubleshooting skills. This test was developed by three teachers who have had experiences of teaching Network Management for over ten years. Ten points was for each multiple choice question; twenty points for the essay question. The total score is 100 points.

- Cognitive style test: the present study adopted a hidden figure test. The total number of questions was 32 and each correct answer scored 1 point. The scoring is that the number of correct answer minuses the number of wrong answer. To adjust the guessing effect, the obtained score was divided by 4 . The total score is 32 .

- The guiding worksheet: based on the scenario of the simulated operation problem, the teacher designed 10 scaffolding guiding questions to assist learners to complete their learning task.

- A simulated operation problem: the teacher used Packet Tracer to design a problem about network concept and troubleshooting. Each student can use the network equipment and set up the network environment on the computer. The learning objective is to troubleshoot the network in the simulated environment.

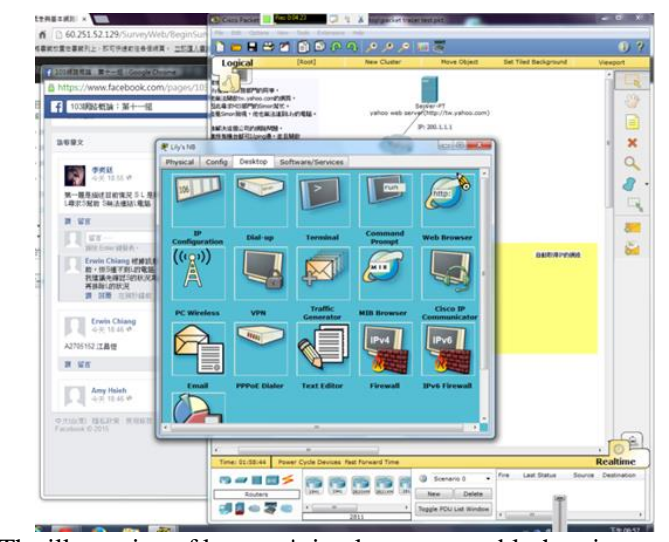

Fig. 3. The illustration of learners' simultaneous troubleshooting practice and discussion by collaborative learning.

\section{RESEARCH RESUlTS}

In week 16, all the participants took the network concept and troubleshooting pretest, and based on the result of the pretest, the learners were divided into groups. The learners took the post test in week 18 after the teaching activity was performed. The total number of the participants was 37, which was divided into 9 groups, each of which consisted of 4 to 5 members. There was no significant difference among the groups, $\mathrm{F}=0.001, p=1.00>.05$. On top of the pretest, in week 16, the cognitive style field-independent/dependent scale, the hidden figure test, was given to the participants. The total score of the hidden figure test ranges from 1 (inclined to field-dependence) to 29 (inclined to field-independence). The learners with different cognitive style were evenly assigned to each group.

Table I shows the results of the paired t-test of pretest and the post-test for all learners, indicating that after engaging in the teaching activity, learners made a significant progress in network concept and troubleshooting, $t=-2.96, p=.005<.01$.

On the basis of the results of the hidden figure test, those whose scores were the top 25\% (9 learners) were classified into field-independent cognitive style; those whose scores were the bottom $25 \%$ ( 9 learners) were classified into 
field-dependent cognitive style. The data from the learners of field-independent and field-dependent cognitive style were then sent to ANCOVA analysis to investigate whether the teaching activity may have different impact on learners with different cognitive style.

TABLE I: PAIRED T-TEST ANALYSIS OF THE PRETEST AND THE POST-TEST FOR ALL LEARNERS

\begin{tabular}{cccccc}
\hline \hline Test Items & Participants & $M$ & $S D$ & $t$ & $p$ \\
\hline pretest & 37 & 50.757 & 21.594 & & \\
post test & 37 & 64.865 & 25.018 & & $p=.005<.01$ \\
\hline \hline
\end{tabular}

The test of the homogeneity of regression slopes of the two cognitive style learners did not achieve significant level, $\mathrm{F}=.94, \mathrm{p}=.35>.05$, indicating that the relationship between pretest and post-test may not differ in the cognitive style groups. Therefore, the data can be sent to the main analysis, ANCOVA. The result of ANCOVA analysis showed that after controlling the effect of the covariate, the pretest, on the dependent variable, post test, the independent variable, the teaching activity, had a significant effect on post test, $\mathrm{F}=4.67$, $p=.047<.050$. This result indicated that the post test of the field-independent learners $\quad(\mathrm{M}=81.33)$ significantly outperform that of the field-dependent learners $(\mathrm{M}=55.56)$.

On the other hand, the learners' discussion in the collaborative problem solving activity was collected, and learners' active discussion and passive responses were calculated. The total number of messages from the 9 field-independent and 9 field-dependent learners in the discussion was 269. Table II shows the descriptive statistics of the number of the messages in the discussion.

TABLE II: DESCRIPTIVE STATISTICS OF THE NUMBER OF MESSAGES IN THE

\begin{tabular}{ccccc}
\hline Group & $\begin{array}{c}\text { Group } \\
\text { Members }\end{array}$ & $\begin{array}{c}\text { Active } \\
\text { Discussion } \\
\text { (Number of } \\
\text { Messages) }\end{array}$ & $\begin{array}{c}\text { Passive } \\
\text { Response } \\
\text { Number of } \\
\text { Messages) }\end{array}$ & $\begin{array}{c}\text { Total } \\
\text { Number of } \\
\text { Message }\end{array}$ \\
\hline $\begin{array}{c}\text { Field-Independent } \\
\begin{array}{c}\text { Style } \\
\text { Field-Dependent } \\
\text { Style }\end{array}\end{array}$ & 9 & $31(35.227 \%)$ & $57(64.773 \%)$ & $88(100 \%)$ \\
\hline \hline
\end{tabular}

\section{DISCUSSION AND CONCLUSION}

The purpose of this study was to investigate the effect of cognitive style on collaborative learning in the field of STEM This study adopted a hidden figure test develop by Messick (1962) and adapted by $\mathrm{Wu}$ (1974) as the measuring instrument to measure learners' cognitive style. The planning of teaching activities followed the procedure of collaborative problem solving strategies, and Facebook was used as a collaborative learning platform in order to observe learners' discussion in the teaching activity. The participants in the present study were the students who took the course of Network Management, and they were asked to complete a task, a simulated operation problem, and troubleshoot the network.

The results of the study showed that after engaging in the learning activities, all the learners make a significant improvement in the post test. This result is consistent with previous studies [2], [3]. Integrating simulated learning tools on the Internet with collaborative problem solving strategies can assist students to learn network and other relevant knowledge.

In addition, it was found that learners with different cognitive style had different performance in the present study. The number of the discussion messages from the learners with field-independent cognitive style was less than that from learners with field-dependent cognitive style in the process of collaborative learning. This result is corresponded with the theory proposed by Witkin (1964). Learners with field-independent cognitive style tend to deal with things in a clear way and to work independently. Furthermore, the behaviors of learners with field-independent cognitive style are more rational or indifferent. In contrast, learners with field-dependent cognitive style not only had more discussion messages, but they were also more active to discuss with one another in the group (67 discussion messages, 37.02\%). That is, the learners with field-dependent cognitive style are better at interaction and more susceptible to the external environment compared with those with field-independent cognitive style. This result is consistent with previous studies [5].

In terms of learning outcome, the learners with field-independent cognitive style outperformed those with field-dependent cognitive style in post test. A possibility is that although in the process of collaborative learning, the learners with field-independent cognitive style had less active discussion messages, they had more passive responses, of which percentage was over $50 \%$ of the total discussion messages. This result indicated that when the learners with field-independent cognitive style assisted others to complete the task, they can also benefit by it.

This study assigned the learners with different cognitive styles evenly to different groups. Although it was found that learners with different cognitive style tend to have different habitual behavior to participate in the activity, i.e., thinking independently or being glad to interact, they can still help one another to learn in a group activity, which was beneficial to improve learning achievement. This result is consistent with Lee (2000) [17].

Finally, this study was a pilot study on the effect of different cognitive style on collaborative learning in the field of STEM. Future studies will continue to investigate the effect and difference of different cognitive style on different teaching scenarios and expect these studies can contribute to assisting students to learn relevant knowledge and skills.

\section{REFERENCES}

[1] P. Doulai, "The role of computer simulation in electric energy systems education," In Tomorrow's Education in Electrical Technologies: Revisited Methods and Tools for Renewed Motivation, European Power Electronics and Drive Association, 2001.

[2] P. C. Lin et al., "Exploring college students' cognitive processing patterns during a collaborative problem-solving teaching activity integrating Facebook discussion and simulation tools," Internet and Higher Education, vol. 22, pp. 51-56, 2014.

[3] H. K. Lu and P. C. Lin, "Effects of interactivity on students' intention to use simulation-based learning tool in computer networking education," in Proc. 3rd International Conference on Next Generation Information Technology (ICNIT 2012), 2012.

[4] H. A. Witkin et al., "Field dependent and field independent cognitive styles and their educational implication," Review of Educational Research, vol. 47, no. 1, pp. 1-64, 1962. 
[5] S. Messick, "Individuality in learning: Implications of cognitive styles and creativity for human development," Learning styles preferences of Korean, Mexican, Armenian-American, and Anglo students in secondary schools. NASSP Bulletin, vol. 81, pp. 103-111, 1962.

[6] C. H. M. Lee et al., "What affect student cognitive style in the development of hypermedia learning system?" Computers \& Education, vol. 45, pp. 1-9, 2005.

[7] J. J. Wu, Hidden Figure Test, Yuan-Liou. Taipei, 1974

[8] R. Riding and E. Sadler-Smith, "Type of instructional material, cognitive style and learning performance," Educational Studies, vol. 18 no. 3, pp. 323-340, 1992.

[9] R. W. Gardner, et al., "A study of individual consistencies in cognitive behavior: Part 4. Psychological issues," Cognitive Control, New York: International Universities Press, 1959

[10] F. Riessman, "Styles of learning," Psychology of Education, New York, 1964.

[11] J. Kagan, "Impulsive and reflective children: Significance of conceptual tempo," Learning and the Educational Process, Chicago: Rand Mcnally, 1965.

[12] A. Gregorc, "A new definition for Individurus," NASSP BuUetin, pp. 20-26, 1977.

[13] L. H. Lin, "The study of the relationship among cognitive style, reasoning performance and problem-solving performance in between aboriginal and non-aboriginal elementary students-an example in regular elementary schools in Tauyen," National Hsinchu University of Education, 2000.

[14] C. M. Liao, "A research of STS teaching and elementary students' cognitive style on science learning," National Taipei University of Education, 2001

[15] S. Y. Chang, A Study on the Relationship between Mathematical Cognitive Styles and Learning Achievement of Junior School Students in Middle School in Kaohsiung, National Kaohsiung Normal University, 2003.

[16] Y. H. Lee, Exploring Group Composition Methods for Cooperative Learning in Web-based Instructional Systems, National Sun Yat-sen University, 2000.

[17] R. M. Gagné, "Learnable aspects of problem solving," Educational Psychologist, vol. 15, no. 2, pp. 84-92, 1980.

[18] R. M. Gagné and L. J. Briggs, Principles of Instructional Design (2nd ed.), New York: Holt, Rinehart \& Winston, 1979

[19] S. Oh and D. H. Jonassen, "Scaffolding online argumentation during problem solving," Journal of Computer Assisted Learning, vol. 23, no. 2, pp. 95-110, 2007.

[20] H. I. Stromso et al., "Content and processes in problem-based learning: A comparison of computer-mediated and face-to-face communication," Journal of Computer Assisted Learning, vol. 23, no. 3, pp. 271-282, 2007.
[21] S. Messick, "Personality consistencies in cognition and creativity," Individuality in Learning, San Francisco, CA: Josey-Bass, pp. 4-33, 1962.

[22] L. M. Nelson, "Instructional-design theoriesand models: A new paradigm of instructional theory," Collaborative Problem Solving, Mahwah, N.J: Erlbaum Associates, 1999.

[23] H. K. Lu and P. C. Lin, "A study of the impact of collaborative problem-solving strategies on students' performance of simulation-based learning - A case of network basic concepts course," International Journal of Information and Education Technology, vol. 7, no. 5, pp. 361-366, 2017.

[24] D. H. Jonassen, Computer in the Classroom: Mindtools for Critical Thinking, Englewood Cliffs, NJ: Prentice-Hall, 1996.

[25] L. Carabaneanu et al., Trends in e-Learning, Tampere Polytechnic, pp. 106-111, 2006

[26] H. C. Liu and I. H. Su, "Learning residential electrical wiring through computer simulation: The impact of computer-based learning environments on student achievement and cognitive load," British Journal of Educational Technology, vol. 42, no. 4, pp. 598-607, 2010

[27] E. Salas et al., "Using simulation-based training to enhance management education," Academy of Management Learning \& Education, vol. 8, pp. 559-573, 2009.

[28] D. Frezzo et al., "Design patterns for learning and assessment: Facilitating the introduction of a complex simulation-based learning environment into a community of instructors," Journal of Science Education and Technology, vol. 19, no. 2, pp. 105-114, 2010.

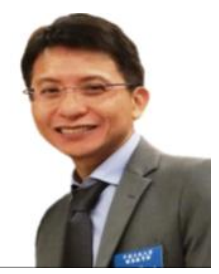

Hsin-Ke Lu is the director of information management (master programs for working learners) and the CEO of School of Continuing Education at Chinese Culture University in Taiwan. $\mathrm{He}$ is the chairman of the Association of Continuing Education of Colleges and Universities in Taiwan and the Chairman of Cisco Networking Academy. His academic interests focus on e-learning, corporate structure, lifelong learning, and information system planning (enterprise architecture).

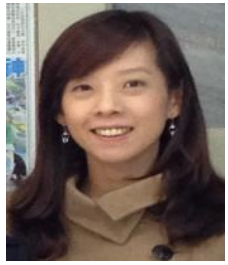

Peng-Chun Lin is an assistant professor of the Department of Information Management in Chinese Culture University. She is also the chief officer of the International Information Certification Cooperation Center at Chinese Culture University in Taiwan. Her academic interests focus on e-learning, network community development, application of simulation learning and recently on optimal network management and corporate structure. 